\title{
Large Forbidden Configurations and Design Theory
}

\author{
R.P. Anstee* \\ Mathematics Department \\ The University of British Columbia \\ Vancouver, B.C. Canada V6T $1 Z 2$ \\ Attila Sali \\ Rényi Institute \\ Budapest, Hungary
}

September 2, 2014

\begin{abstract}
Let forb $(m, F)$ denote the maximum number of columns possible in a $(0,1)$ matrix $A$ that has no repeated columns and has no submatrix which is a row and column permutation of $F$. We consider cases where the configuration $F$ has a number of columns that grows with $m$. For a $k \times \ell$ matrix $G$, define $s \cdot G$ to be the concatenation of $s$ copies of $G$. In a number of cases we determine forb $\left(m, m^{\alpha} \cdot G\right)$ is $\Theta\left(m^{k+\alpha}\right)$. Results of Keevash on the existence of designs provide constructions that provide asymptotic lower bounds. An induction idea of Anstee and Lu is useful in obtaining upper bounds.
\end{abstract}

Keywords: extremal set theory, BIBD, $t$-designs, $(0,1)$-matrices, multiset, forbidden configurations, trace, subhypergraph.

\section{Introduction}

We first give the matrix notation for our extremal problems. Define a matrix to be simple if it is a $(0,1)$-matrix with no repeated columns. Define that $F$ is a configuration in $A$ (denoted $F \prec A$ ) if there is a submatrix of $A$ which is a row and column permutation of

${ }^{*}$ Research supported in part by NSERC 
$F$. If we think of a simple matrix as the element-set incidence matrix of a set system, then a configuration corresponds to the trace. Define:

$$
\begin{gathered}
\operatorname{Avoid}(m, F)=\{A: A \text { is } m \text {-rowed simple, } F \nprec A\}, \\
\operatorname{forb}(m, F)=\max _{A}\{\|A\|: A \in \operatorname{Avoid}(m, F)\} .
\end{gathered}
$$

For two matrices $X, Y$ on the same number of rows let $[X \mid Y]$ denote the concatenation of $X$ and $Y$. Let $s \cdot F=[F|F| \cdots \mid F]$ be the concatenation of $s$ copies of $F$. Considering $s$ to be a growing function of $m$ and computing forb $(m, s \cdot F)$ is the focus of this paper. In this paper we typically choose $s=m^{\alpha}$. Think of $m^{\alpha}$ as an 'honourary integer'. Of course it would be correct to write $\left\lfloor m^{\alpha}\right\rfloor$ instead but this is not done to keep the presentation simpler. Let $K_{k}$ denote the $k \times 2^{k}$ matrix of all $(0,1)$-columns on $k$ rows and let $K_{k}^{\ell}$ denote the $k \times\left(\begin{array}{l}k \\ l\end{array}\right)$ matrix of all possible $(0,1)$-columns of column sum $\ell$ on $k$ rows. Let $\mathbf{1}_{k}$ (respectively $\mathbf{0}_{k}$ ) denote the $k \times 1$ columns of 1 's (resp. 0's).

Computing forb $\left(m, s \cdot \mathbf{1}_{k}\right)$ requires some results. Recent breakthrough results of Peter Keevash give the existence of simple designs that are useful to provide lower bounds. Given parameters $t, m, k, \lambda$, a $t$ - $(m, k, \lambda)$ design $\mathcal{D}$ is a multiset of subsets in $\left(\begin{array}{c}{[m]} \\ k\end{array}\right)$ such that for each $S \in\left(\begin{array}{c}{[m]} \\ t\end{array}\right)$ there are exactly $\lambda$ blocks $B \in \mathcal{D}$ containing $S$. A $t$ - $(m, k, \lambda)$ design $\mathcal{D}$ is simple if $\mathcal{D}$ is a set (i.e. no repeated blocks). A $t$ - $(m, k, \lambda)$ design $\mathcal{D}$ can be encoded as a $m \times \frac{\lambda}{\left(\begin{array}{c}k \\ t\end{array}\right)}\left(\begin{array}{c}m \\ t\end{array}\right)$ element-block incidence matrix $A$. Then $A$ is simple if and only if the design is simple. Then each $t$-tuple of rows contains $\lambda \cdot \mathbf{1}_{t}$ and $A \in \operatorname{Avoid}\left(m,(\lambda+1) \cdot \mathbf{1}_{t}\right)$. A quite complete result for simple triple systems had already been given.

Theorem 1.1 (Dehon [10]) Let $m, \lambda$ be given. Assume $m \geq \lambda+2$ and $m \equiv 1,3(\bmod 6)$. Then there exists a simple $2-(m, 3, \lambda)$ design.

Corollary 1.2 Let $\lambda$ be given with $\lambda \leq m-2$. Assume $m \equiv 1,3(\bmod 6)$. Then forb $\left(m,(\lambda+2) \cdot \mathbf{1}_{2}\right)=\left(\begin{array}{c}m \\ 0\end{array}\right)+\left(\begin{array}{c}m \\ 1\end{array}\right)+\left(\begin{array}{c}m \\ 2\end{array}\right)+\frac{\lambda}{3}\left(\begin{array}{c}m \\ 2\end{array}\right)$.

Proof: Let $M$ be the element-block incidence matrix of a simple $2-(m, 3, \lambda)$ design. Then $M \in \operatorname{Avoid}\left(m,(\lambda+1) \cdot \mathbf{1}_{2}\right)$. Let $B=\left[K_{m}^{0}\left|K_{m}^{1}\right| K_{m}^{2} \mid M\right]$. Then $B \in \operatorname{Avoid}(m,(\lambda+$ $\left.2) \cdot \mathbf{1}_{2}\right)$.

Corollary 1.3 Let $0<\alpha \leq 1$ be given. Then forb $\left(m, m^{\alpha} \cdot \mathbf{1}_{2}\right)$ is $\Theta\left(m^{2+\alpha}\right)$.

Proof: Let $\lambda=m^{\alpha}-2$. We apply the construction of Conjecture 1.2 when the divisibility conditions for $m$ are satisfied. When $m$ does not satisfy the divisibilty conditions then choose the largest $m^{\prime}<m$ that does satisfy divisibility conditions. Again find $M$ as the $m^{\prime} \times \frac{m^{\alpha}-2}{3}\left(\begin{array}{c}m^{\prime} \\ 2\end{array}\right)$ element-block incidence matrix of a $2-(m, 3, \lambda)$ design. Let $B=\left[K_{m^{\prime}}^{0}\left|K_{m^{\prime}}^{1}\right| K_{m^{\prime}}^{2} \mid M\right]$. Then $B \in \operatorname{Avoid}\left(m^{\prime},(\lambda+2) \cdot \mathbf{1}_{2}\right)$. Now add $m-m^{\prime}$ rows of 1's to finish the construction of a matrix $A \in \operatorname{Avoid}\left(m, m^{\alpha}\right)$ with $\|A\|$ being 
$\left(\begin{array}{c}m^{\prime} \\ 0\end{array}\right)+\left(\begin{array}{c}m^{\prime} \\ 1\end{array}\right)+\left(\begin{array}{c}m^{\prime} \\ 2\end{array}\right)+\frac{m^{\alpha}-2}{3}\left(\begin{array}{c}m^{\prime} \\ 2\end{array}\right)$ This is $\Theta\left(\left(m^{\prime}\right)^{2} m^{\alpha}\right)$ and since $m^{\prime}>m / c$ for some constant $c>0$ (we need only a weak estimate) we have that $\|A\|$ is $\Theta\left(m^{2+\alpha}\right)$.

The following remarkable existence theorem appears as Theorem 6.6 in [12]. Note that the divisibility conditions are what is required for a $t-(m, k, 1)$ simple design. Keevash has a slightly different theorem for larger $\lambda$ which would have slightly weaker divisibility conditions. A surprising part of this result is that the design is simple and hence can be used as a construction for our problems.

Theorem 1.4 (Keevash [12]) Let $1 / m \ll \theta \ll 1 / k \leq 1 /(t+1)$ and $\theta \ll 1$. Suppose that $\left(\begin{array}{c}k-i \\ t-i\end{array}\right)$ divides $\left(\begin{array}{c}m-i \\ t-i\end{array}\right)$ for $0 \leq i \leq r-1$. Then there exists a $t-(m, k, \lambda)$ simple design for $\lambda \leq \theta m^{k-t}$.

Note that $1 \leq \lambda \leq\left(\begin{array}{c}m \\ k\end{array}\right)\left(\begin{array}{c}k \\ t\end{array}\right) /\left(\begin{array}{c}m \\ t\end{array}\right)$ and so this covers a fraction $\theta$ of the possible range for $\lambda$. Baranyai's Theorem when applied to decompose the complete $r$-uniform hypergraph into sets of disjoint edges, each set of size $m / r$, yields the following result that is helpful for $t=1$.

Theorem 1.5 [9] Let $m, r$ be given with $r$ divides $m$. Then we can create $\left(\begin{array}{c}m-1 \\ r-1\end{array}\right)$ matrices $M_{1}, M_{2}, \ldots, M_{\left(\begin{array}{c}m-1 \\ r-1\end{array}\right)}$ such that $K_{m}^{r}=\left[M_{1}\left|M_{2}\right| \cdots \mid M_{\left(\begin{array}{c}m-1 \\ r-1\end{array}\right)}\right]$ where each $M_{i}$ consists of $m / r$ columns of sum $r$ and each row sum is 1.

A warmup is the following exact result.

Proposition 1.6 Let $t \geq 1$ be given. Then forb $\left(m,\left(1+\sum_{i=t}^{r}\left(\begin{array}{c}m-t \\ i-t\end{array}\right)\right) \cdot \mathbf{1}_{t}\right)=\sum_{i=0}^{r}\left(\begin{array}{c}m \\ i\end{array}\right)$.

Proof: We have the construction $\left[K_{m}^{0} K_{m}^{1} \cdots K_{m}^{r}\right]$ which has no configuration $(m+$ $\left.\sum_{i=t}^{r}\left(\begin{array}{c}m-2 \\ i-t\end{array}\right)\right) \cdot \mathbf{1}_{t}$. The upper bound follows by the pigeonhole bound (1). We would preferentially choose the columns of smallest column sum. It is easy to verify that $K_{m}^{i}$ contains $\left(\begin{array}{c}m-t \\ i-t\end{array}\right) \cdot \mathbf{1}_{t}$ in each $i$-tuple of rows but $\left(1+\left(\begin{array}{c}m-t \\ i-t\end{array}\right)\right) \cdot \mathbf{1}_{t} \nprec K_{m}^{i}$.

Given parameters $t, m, k, \lambda$, define a $t$ - $(m, k, \lambda)$ packing $\mathcal{P}$ to be a set of subsets in $\left(\begin{array}{c}{[m]} \\ k\end{array}\right)$ such that for each $S \in\left(\begin{array}{c}{[m]} \\ t\end{array}\right)$ there are at most $\lambda$ blocks $B \in \mathcal{D}$ containing $S$. For this paper repeated blocks are not allowed. Such a packing $\mathcal{P}$, when encoded by its element-block incidence matrix, yields a $m \times|\mathcal{P}|$ matrix in $\operatorname{Avoid}\left(m,(\lambda+1) \cdot \mathbf{1}_{t}\right)$.

Theorem 1.7 Let $t \geq 1$ be given. There is a $t-\left(m, k, m^{\alpha}\right)$ packing $\mathcal{P}$ with $|\mathcal{P}|$ being $\Theta\left(m^{t+\alpha}\right)$ and so forb $\left(m, m^{\alpha} \cdot \mathbf{1}_{t}\right)$ is $\Theta\left(m^{t+\alpha}\right)$.

Proof of Theorem 1.7: Let $A \in \operatorname{Avoid}\left(m, s \cdot \mathbf{1}_{t}\right)$. Let $a_{i}$ be the number of columns of sum $i$. The pigeonhole bound becomes

$$
\|A\| \leq \sum_{i=t}^{m} a_{i}\left(\begin{array}{l}
i \\
t
\end{array}\right) \leq(s-1)\left(\begin{array}{c}
m \\
t
\end{array}\right)
$$


Of course we also have $a_{i} \leq\left(\begin{array}{c}m \\ i\end{array}\right)$.

First consider $t=1$ and apply Baranyai's Theorem 1.5. Determine a value $r$ so that

$$
\left(1+\sum_{i=t}^{r}\left(\begin{array}{c}
m-1 \\
i-1
\end{array}\right)\right)<m^{\alpha}<\left(1+\sum_{i=t}^{r+1}\left(\begin{array}{c}
m-1 \\
i-1
\end{array}\right)\right) .
$$

Assume $r$ divides $m$. Then let $v=m^{\alpha}-\left(1+\sum_{i=t}^{r}\left(\begin{array}{c}m-1 \\ i-1\end{array}\right)\right)$ and use Theorem 1.5 to find $v$ 'perfect matchings', namely $M_{1}, M_{2}, \ldots, M_{v}$ and then $\left[K_{m}^{0} K_{m}^{1} \cdots K_{m}^{r} M_{0} M_{1} \cdots M_{v}\right] \in$ $\operatorname{Avoid}\left(m, m^{\alpha} \cdot \mathbf{1}_{t}\right)$ with $\Theta\left(m^{t+\alpha}\right)$ columns. The asymptotics follow for cases where $r$ does not divide $m$.

For $t \geq 2$ follow a similar argument but now using Theorem 1.4. Determine a value $r$ with

$$
\left(1+\sum_{i=t}^{r}\left(\begin{array}{c}
m-t \\
i-t
\end{array}\right)\right)<m^{\alpha}<\left(1+\sum_{i=t}^{r+1}\left(\begin{array}{c}
m-t \\
i-t
\end{array}\right)\right) .
$$

Let $s=m^{\alpha}-\left(1+\sum_{i=t}^{r}\left(\begin{array}{c}m-t \\ i-t\end{array}\right)\right)$. Then we form $A$ from the concatenation of $\left[K_{m}^{0} K_{m}^{1} \cdots K_{m}^{r}\right]$ and the element-block incidence matrix of an $t-(m, r+1, s)$ packing that has $\Theta\left(\mathrm{sm}^{r}\right)$ columns. The result will have $\Theta\left(m^{t+\alpha}\right)$ columns where the constant will depend on $\alpha, t$. In particular the constant would shrink with larger $\alpha$.

We use this result extensively in Section 3 to provide lower bounds. The results given there consider a fixed simple configuration $F$ and then a configuration $F(s)$ obtained from $F$ by repeating certain selected columns $s$ times. The cases considered have already had the asymptotic growth of forb $(m, F(s))$ determined for fixed $s$. This paper considers $s=m^{\alpha}$ times. The following basic result for $F=K_{k}$ is extended to a result for $F=m^{\alpha} \cdot K_{k}$.

Theorem 1.8 (Sauer[13], Perles and Shelah[14], Vapnik and Chervonenkis[15])

$$
f \circ r b\left(m, K_{k}\right)=\left(\begin{array}{c}
m \\
k-1
\end{array}\right)+\left(\begin{array}{c}
m \\
k-2
\end{array}\right)+\cdots+\left(\begin{array}{c}
m \\
0
\end{array}\right) .
$$

Theorem 1.9 Assume $k, \alpha$ are given and $m^{\alpha} \geq 2$. Then forb $\left(m, m^{\alpha} \cdot K_{k}\right)$ is $\Theta\left(m^{k+\alpha}\right)$.

Proof: The lower bound follows from Theorem 1.7 since $\mathbf{1}_{k} \prec K_{k}$. The upper bound follows using forb $\left(m, m^{\alpha} \cdot K_{k}\right)=\operatorname{forb}\left(m, m^{\alpha} \cdot \mathbf{1}_{k}\right)$ (Theorem 4.4 in [6]).

\section{$2 \quad$ New Induction}

We consider a new form of the standard induction [1] for forbidden configurations [2]. For a matrix $A$, let $\mu(\mathbf{x}, A)$ denote the multiplicity of $\mathbf{x}$ as a column of $A$. We say $A$ is $(s-1)$-simple if $\mu(\mathbf{x}, A) \leq s-1$ for all columns $\mathbf{x}$. We define Let $F$ be a matrix with 
maximum column multiplicity $t$. Thus $F \prec t \cdot \operatorname{supp}(F)$. Let $A \in \operatorname{Avoid}(m, F, t-1)$. Assume $\|A\|=\operatorname{forb}(m, \mathcal{F}, t-1)$. Given a row $r$ we permute rows and columns of $A$ to obtain

$$
A=\operatorname{row} r \rightarrow\left[\begin{array}{cc}
00 \cdots 0 & 11 \cdots 1 \\
G & H
\end{array}\right]
$$

Now $\mu(\alpha, G) \leq t$ and $\mu(\alpha, H) \leq t$. For those $\alpha$ for which $\mu(\alpha,[G H])>t$, let $C$ be formed with $\mu(\alpha, C)=\min \{\mu(\alpha, G), \mu(\alpha, H)\}$. We rewrite our decomposition of $A$ as follows:

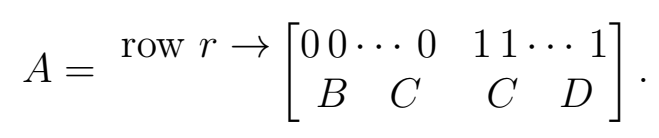

Then we deduce that $[B C D]$ and $C$ are both $(t-1)$-simple. The former follows from $\mu(\alpha,[B C D])=\mu(\alpha, G)+\mu(\alpha, H)-\min \{\mu(\alpha, G), \mu(\alpha, H)\} \leq t$. We have that $F \nprec[B C D]$ for $F \in \mathcal{F}$. Since each column $\alpha$ of $C$ has $\mu(\alpha,[G H]) \geq t$, we deduce that $\operatorname{supp}(F) \nprec C$ for each $F \in \mathcal{F}$. One induction on $m$ becomes:

$$
\begin{aligned}
& \operatorname{forb}(m, \mathcal{F}, t-1)=\|A\|=\|[B C D]\|+\|C\| \\
& \quad \leq \operatorname{forb}(m-1, \mathcal{F}, t-1)+(t-1) \cdot \operatorname{forb}(m-1,\{\operatorname{supp}(F): F \in \mathcal{F}\}) .
\end{aligned}
$$

This is a simplified version of what appears in [1] only using the fact that $C \in \operatorname{Avoid}(m-$ $1, \operatorname{supp}(F))$ and keeping track of the various $C$ 's as they are produced.

Lemma 2.1 Let $m, s$ be given. Let $\mathcal{F}$ consist of simple matrices. Then

$$
\operatorname{forb}(m,\{s \cdot F: F \in \mathcal{F}\}) \leq \sum_{i=1}^{m}(s-1) \cdot \operatorname{forb}(m-i, \mathcal{F}) .
$$

Proof: Apply induction using forb $(m, s \cdot F) \leq$ forb $(m, s \cdot F, t)$ so that forb $(m,\{s \cdot F$ : $F \in \mathcal{F}\}) \leq \operatorname{forb}(m,\{s \cdot F: F \in \mathcal{F}\}, s)$. We keep $s$ is fixed during the course of the induction but $s$ can be a function of $m$, e.g. $s=m$ is possible.

\section{$3 \quad$ Large Configurations}

For convenience when dealing with asymptotics we take $s=m^{\alpha}$. When we say $f(m)$ is $\Theta\left(m^{2+\alpha}\right)$ for a given $\alpha$ then we are allowing a constant $c_{\alpha}$ with $f(m) \leq c_{\alpha} m^{2+\alpha}$.

Theorem 3.1 Let $\mathbf{1}_{k} \prec F$ and assume forb $(m, F)$ is $\Theta\left(m^{k-1}\right)$. Then for $2 \leq s$, forb $(m, s \cdot F)$ is $\Theta\left(s \cdot m^{k}\right)$. Thus forb $\left(m, m^{\alpha} \cdot F\right)$ is $\Theta\left(m^{k+\alpha}\right)$. 
Proof: The lower bound follows Lemma 1.7. The upper bound follows from (6). Note that if there is a constant $c$ so that for all $i \geq 1$, forb $(i, F) \leq c \cdot \sum_{i=0}^{k-1}\left(\begin{array}{l}i \\ j\end{array}\right)$, then $\sum_{i=1}^{m} \operatorname{forb}(i, F) \leq c \cdot \sum_{i=0}^{k}\left(\begin{array}{c}m \\ j\end{array}\right)$.

There are many $F$ with the desired property [2]. Consider the following configurations.

$$
F_{0}=\left[\begin{array}{l}
1 \\
0
\end{array}\right] \quad F_{1}=\left[\begin{array}{ll}
1 & 1 \\
1 & 0 \\
0 & 1 \\
0 & 0
\end{array}\right] \quad F_{2}=\left[\begin{array}{lll}
1 & 1 & 1 \\
1 & 1 & 0 \\
1 & 0 & 1 \\
0 & 1 & 0 \\
0 & 0 & 1 \\
0 & 0 & 0
\end{array}\right]
$$

Theorem 3.2 [2] $\operatorname{forb}\left(m, F_{0}\right)=2$.

Theorem $3.3[3] \operatorname{forb}\left(m, F_{1}\right)=4 m-4$.

Theorem 3.4 [7] forb $\left(m, F_{2}\right)$ is $\Theta\left(m^{2}\right)$.

The following are sample large forbidden configuration theorems.

Corollary 3.5 Let $\alpha$ be given. Then forb $\left(m, m^{\alpha} \cdot F_{0}\right)$ is $O\left(m^{1+\alpha}\right)$ and forb $\left(m, m^{\alpha} \cdot F_{1}\right)$ is $O\left(m^{2+\alpha}\right)$ and forb $\left(m, m^{\alpha} \cdot F_{2}\right)$ is $O\left(m^{3+\alpha}\right)$.

Proof: The lower bounds arise from Theorem 1.7 and the upper bounds follow by induction from (6) using the bounds of Theorems 3.2,3.3 and 3.4 as appropriate.

There are other families of examples. Let $I_{k}$ denote the $k \times k$ identity matrix, let $I_{k}^{c}$ denote the $(0,1)$-complement of $I_{k}$ and let $T_{k}$ denote the upper triangular matrix with the $(i, j)$ entry equal 1 if and only if $i \leq j$. The following is the essential way to obtain a constant bound:

Theorem 3.6 [8] Let $k$ be given. There is a constant $c_{k}$ with forb $\left(m,\left\{I_{k}, I_{k}^{c}, T_{k}\right\}\right)=c_{k}$.

If, in the following result, $m^{\alpha}$ is replaced by a fixed $s \geq 2$, the bound of $\Theta(m)$ was established in [1].

Theorem 3.7 Let $\alpha$ be given with $m^{\alpha} \geq 2$. Then forb $\left(m,\left\{m^{\alpha} \cdot I_{k}, m^{\alpha} \cdot I_{k}^{c}, m^{\alpha} \cdot T_{k}\right)\right.$ is $\Theta\left(m^{1+\alpha}\right)$.

Proof: Note that $F_{0} \prec I_{k}, F_{0} \prec I_{k}^{c}$ and $F_{0} \prec T_{k}$. Thus forb $\left(m,\left\{m^{\alpha} \cdot I_{k}, m^{\alpha} \cdot I_{k}^{c}, m^{\alpha} \cdot T_{k}\right) \geq\right.$ forb $\left(m, m^{\alpha} \cdot F_{0}\right) \geq$ forb $\left(m, m^{\alpha} \cdot \mathbf{1}_{1}\right)$. The lower bound follows from Theorem 1.7 when $t=1$. The upper bound follows from the induction (6) and Theorem 3.6. 
For a set $S \subseteq[\mathrm{m}]$, define $\left.A\right|_{S}$ to be the submatrix of $A$ consisting of the rows of $S$. Let $B$ be a $k \times(k+1)$ simple matrix with one column of each column sum and let $F_{B}(s)=\left[K_{k} \mid(s-1) \cdot\left[K_{k} \backslash B\right]\right]$, where the notation $C \backslash D$ refer to the matrix contained from $C$ by deleting all columns in $D$. Let $D_{12}(k)$ be the $k$-rowed simple matrix of all columns which do not have $\mathbf{1}_{2}$ in rows 1,2 and also does not have the column of 0's. Let $F_{12}(s)=\left[\mathbf{0}_{k} \mid s \cdot D_{12}(k]\right.$. Note that in either matrix there is a column of multiplicity 1 and not $s$.

The major result of [4] yields that $F_{B}(s)$ and $F_{12}(s)$ are the maximal $k$-rowed matrices which have forb being $O\left(\mathrm{~m}^{k-1}\right)$. Here maximal means that adding a column not already present $s$ times will result in a configurations with forb being $\Omega\left(m^{k}\right)$. The following lemma is helpful.

Lemma 3.8 (Lemma 4.4 in [5]) Let $A, k$ and $u$ be given and assume that $\mathcal{S}$ denotes those $k$-sets of rows $S$ of $A$ for which $\left.A\right|_{S}$ has at least two different $k \times 1$ columns $\alpha, \beta$ with $\mu\left(\alpha,\left.A\right|_{S}\right)<u$ and $\mu\left(\beta,\left.A\right|_{S}\right)<u$. We can delete $O\left(m^{k-1}\right)$ columns from $A$ to obtain $A^{\prime}$ so that for each $S \in \mathcal{S}$, there is some column not present in $\left.A\right|_{S}$.

In fact there exists a set of at most

$$
2 u\left(\left(\begin{array}{c}
m \\
k-1
\end{array}\right)+\left(\begin{array}{c}
m \\
k-2
\end{array}\right)+\cdots+\left(\begin{array}{c}
m \\
0
\end{array}\right)\right)
$$

columns to delete from $A$ to obtain $A^{\prime}$. We use this with $u=m^{\alpha}$. The following proof is in [4].

Theorem 3.9 Let $k$ be given and $B$ be a given $k \times(k+1)$ simple matrix with one column of each column sum. Then forb $\left(m, F_{B}\left(m^{\alpha}\right)\right)$ is $\Theta\left(m^{k-1+\alpha}\right)$.

Proof: The lower bound follows from Theorem 1.7 with $k$ replaced by $k-1$. The upper bound follows from considering $A \in \operatorname{Avoid}\left(m, F_{B}\left(m^{\alpha}\right)\right)$ and noting that for each $k$-set of rows either there is some column not present in $\left.A\right|_{S}$ or there are two $k \times 1$ columns $\alpha, \beta$ with $\mu\left(\alpha,\left.A\right|_{S}\right)<m^{\alpha}$ and $\mu\left(\beta,\left.A\right|_{S}\right)<m^{\alpha}$. Now by Lemma 3.8 with $u=m^{\alpha}$, we obtain a matrix $A^{\prime}$ where for every $k$-set $S$ there is some column not present in $\left.A\right|_{S}$. Thus $A^{\prime} \in \operatorname{Avoid}\left(m, K_{k}\right)$ and Theorem 1.8 completes the proof.

The proof for $F_{12}\left(m^{\alpha}\right)$ again follows the proof in [5].

Theorem 3.10 Let $k$ be given. Then forb $\left(m, F_{12}\left(m^{\alpha}\right)\right)$ is $\Theta\left(m^{k-1+\alpha}\right)$.

Proof: The lower bound follows from Theorem 1.7 with $k$ replaced by $k-1$. The upper bound follows from considering $A \in \operatorname{Avoid}\left(m, F_{12}\left(m^{\alpha}\right)\right)$. Section 2 in [5] outlines the proof. Let $\mathcal{S}$ denotes those $k$-sets of rows $S$ of $A$ for which $\left.A\right|_{S}$ has at least two different $k \times 1$ columns with $\mu\left(\alpha,\left.A\right|_{S}\right)<m^{\alpha}$ and $\mu\left(\beta,\left.A\right|_{S}\right)<m^{\alpha}$. By Lemma 3.8, one can delete at most $O\left(m^{k-1+\alpha}\right)$ columns from $A$ to obtain $A^{\prime}$ where now $\mu\left(\alpha,\left.A\right|_{S}\right)=\mu\left(\beta,\left.A\right|_{S}\right)=0$. We delete a further $O\left(m^{k-1+\alpha}\right)$ columns from $A^{\prime}$ to obtain $A^{\prime \prime}$ which have no "violated 
inner implications' and then deleting a further $O\left(m^{k-1+\alpha}\right)$ columns from $A^{\prime \prime}$ to obtain $A^{\prime \prime \prime}$ where there are only a restricted number of 'violated outer implications' and finally deleting a further $O\left(m^{k-1+\alpha}\right)$ columns from $A^{\prime \prime \prime}$ to obtain $A^{\prime \prime \prime \prime} \in \operatorname{Avoid}\left(m, K_{k}\right)$ with no 'violated outer implications'. Then by Theorem 1.8, $\left\|A^{\prime \prime \prime \prime}\right\|$ is $O\left(m^{k-1}\right)$.

An open problem is to do the same analysis for the following configuration named the 'chestnut'. Define

$$
F_{3}=\left[\begin{array}{ll}
1 & 0 \\
1 & 0 \\
0 & 1 \\
0 & 1
\end{array}\right] .
$$

The following bound is implictly given in [11].

Theorem 3.11 [11] forb $\left(m, F_{3}\right)=\left(\begin{array}{c}m \\ 2\end{array}\right)+2 m-1$

In [1], an induction for the chestnut considers forb $\left(m, t \cdot F_{3}\right) \leq \sum_{i=1}^{m-1}$ forb $\left(i,\left\{F_{3}, t\right.\right.$. $\left.\left.\left[\mathbf{1}_{1} \mathbf{0}_{2} \mid \mathbf{0}_{1} \mathbf{1}_{2}\right]\right\}\right)$ for fixed $t$. It is shown that forb $\left(m,\left\{F_{3}, t \cdot\left[\mathbf{1}_{1} \mathbf{0}_{2} \mid \mathbf{0}_{1} \mathbf{1}_{2}\right]\right\}\right) \leq c t m$ for some constant $c$. Combining Theorem 3.11 we have that forb $\left(m,\left\{F_{3}, t \cdot\left[\mathbf{1}_{1} \mathbf{0}_{2} \mid \mathbf{0}_{1} \mathbf{1}_{2}\right]\right\}\right) \leq$ $\min \left\{c t m,\left(\begin{array}{c}m \\ 2\end{array}\right)+2 m-1\right\}$. Induction (6) yields forb $\left(m, t \cdot F_{3}\right) \leq t m \cdot \min \left\{m^{2}, c t m\right\}$. For fixed $t$, this yields $\Theta\left(m^{2}\right)$ for which we have a matching construction. For larger $t=m^{\alpha}$, the lower bound is $\Omega\left(m^{2+\alpha}\right)$ by Lemma 1.7 and the upper bound is $O\left(m^{\min \{2+2 \alpha, 3+\alpha\}}\right)$. What is the truth?

Problem 3.12 Determine forb $\left(m, m^{\alpha} \cdot F_{3}\right)$.

Another simple related problem is $K_{4}^{2}$ for which it is known that forb $\left(m, K_{4}^{2}\right)$ is $\Theta\left(m^{3}\right)$ and $\operatorname{forb}\left(m, 2 \cdot K_{4}^{2}\right)$ is $\Theta\left(m^{4}\right)$. What is forb $\left(m, m^{\alpha} \cdot K_{4}^{2}\right)$ ? The lower bound is only $\Omega\left(m^{2+\alpha}\right)$ by Lemma 1.7. There are other reasonable problems for large forbidden configurations such as forb $\left(m, s \cdot \mathbf{1}_{\sqrt{m}}\right)$. For $s=1$, the bound is easy.

\section{References}

[1] R.P. Anstee and Linyuan Lu, Repeated columns and an old chestnut, Elec. J. of Combinatorics 20 (2013), P2, 11pp.

[2] R.P. Anstee, A Survey of forbidden configurations results, Elec. J. of Combinatorics 20 (2013), DS20, 56pp.

[3] R.P. Anstee, F. Barekat, A, Sali, , Small Forbidden Configurations V: Exact Bounds for 4x2 cases, Studia Sci. Math. Hun., 48(2011), 1-22.

[4] R.P. Anstee and B. Fleming, Two refinements of the bound of Sauer, Perles and Shelah and Vapnik and Chervonenkis, Discrete Mathematics 310 (2010), 33183323. 
[5] R.P. Anstee and B. Fleming, Linear algebra methods for forbidden configurations, Combinatorica 31 (2011), 119.

[6] R.P. Anstee, A forbidden configuration theorem of Alon, J. Combin. Theory Ser AMath. 47 (1988), 16-27.

[7] R.P. Anstee, M. Raggi, A, Sali, Forbidden Configurations: Boundary Cases, European J. Combin, 35(2014), 51-66.

[8] J. Balogh, B. Bollobás, Unavoidable Traces of Set Systems, Combinatorica, 25 (2005), 633-643.

[9] Zs. Baranyai, On the factorization of the complete uniform hypergraph, Infinite and Finite Sets, Proc. Coll. Keszthely, 1973, Hajnal, A.; Rado, R.; Ss, V. T. eds., Colloquia Math. Soc. Jnos Bolyai 10, North-Holland, pp. 91107.

[10] Michel Dehon, On the existence of 2-designs $S_{\lambda}(2,3, v)$ without repeated blocks, Discrete Math. 43(1983), 155-171.

[11] P. Frankl, Z. Füredi, and J. Pach, Bounding one-way differences, Graphs and Combinatorics 3 (1987), 341-347.

[12] P. Keevash, The Existence of Designs, ArXiv: 1401.3665

[13] N. Sauer, On the density of families of sets, J. Combin. Th. Ser A 13(1972), 145-147.

[14] S. Shelah, A combinatorial problem: Stability and order for models and theories in infinitary languages, Pac. J. Math. 4(1972), 247-261.

[15] V.N. Vapnik and A.Ya. Chervonenkis, On the uniform convergence of relative frequencies of events to their probabilities, Th. Prob. and Applics. 16(1971), 264-280. 